\title{
Environmental Education for Sustainable Development
}

\author{
Arkady Ursul \\ Doctor of Philosophical Sciences, Professor Lomonosov Moscow State University \\ (Moscow, Russia) \\ E-mail: ursul-ad@mail.ru \\ ORCID: 0000-0003-2643-5558

\section{Tatiana Ursul} \\ Doctor of Philosophical Sciences, \\ Professor National Research Technological University "MISiS" \\ (Moscow, Russia) \\ E-mail: ursult@mail.ru \\ ORCID: 0000-0002-4520-9487
}

The authors believes that by continuing the further roll-out of education for sustainable development (ESD), however, need to start implementing a new strategy for global educational process to accelerate and expand the movement towards "global sustainability". It is shown that the environmental component (and corresponding model) of education currently prevalent in the form of education, which is now called ESD, but this is only the beginning of a formation of a new systemic model of education for sustainable development. For the ecological component of ESD has already added the vision of ESD as a leading education and interpretation education based on the concept of "security through sustainable development."

Keywords: anticipated education, education for sustainable development, environmental education, security, sustainable development, futurization

Received: May 28, 2017; accepted: December 29, 2017

Future Human Image, Volume 9, 2018:

DOI: $10.29202 /$ fhi $/ 9 / 12$

\section{Introduction}

In modern education, there are too many different contradictions, which are known to almost everyone who somehow dealt or deals with education (and that has all socialized individuals). In addition, we can hardly find a person who could not be able to offer his "recipe"

(C) Ursul, Arkady, 2018

(C) Ursul, Tatiana, 2018 
for a way out of that deplorable state in which the world educational system is "preserved". Nowadays people write a lot about the need of a fundamental change in strategy of the world education, but constructive suggestions are often reduced to "patching up the holes" in the guise of modernization. Meanwhile, education must become a truly innovative process and one of the main factors in the creation of that new society that will help mankind to survive the approaching global cataclysms and especially — the threatening anthropoecological catastrophe. Rapidly changing socio-natural dynamics dictates new requirements to the educational process in order to provide innovative advanced nature of training of students both in terms of the satisfaction of the needs of society (including strategic ones) and needs of the educational process resulting from them. It is clear that from the modernization of education that transforms education in accordance with the requirements of modernity, it is necessary to transit to its futurization, to the satisfaction of the leading needs of present and all the more of future generations.

Already now it is necessary to anticipate new opportunities and the leading needs of social development, and not just those which occur at this moment in time. Meanwhile, the traditionally established situation in the world education (including, of course, Russian education) indicates a clear commitment of official structures of education to a stable orientation to the past, to the transmission and mastering during training pretty outdated information. And although in the official documents of the modernization of education it is still possible to see the tendency of their creators to some innovations, in the adopted state educational standards the already outdated knowledge and values are yet stressed.

For all scientists and educators it is well known that textbooks and manuals are published on the basis of scientific material which is well proven by practice and substantiated by theories. In addition, this is closely related to the traditionally-translational understanding of education as the transfer of knowledge, skills, values, culture to current generations from previous ones. Actually here we speak about (whether we want to admit it or not) the motion of obsolete knowledge and values in the educational process that are only slightly can be used in future human activity. Hypothesis and assumptions rarely get into teaching materials and therefore actually inform about the past of the scientific search, about the things that have already been established in science, but are still far from its front edge of exploratory activity. It is no coincidence that scientific knowledge about sustainable development is outside of the area of education in school and universities, because the current Russian "managers of education" have not received this knowledge in the process of their learning and by virtue of a narrow worldview, which does not extend beyond market horizons, do not think about the future generations and their needs in the strategic perspective.

Meanwhile, the upcoming transition to sustainable development provides the opportunity for all humanity to move to a wider implementation of the approach to education, which is considered in the Platonic sense, as the shaping in accordance with the ideal (which is described in the article by Oleg Bazaluk (Bazaluk, 2017; 2018).

\section{The Futurization of Human Needs}

In the book "Our Common Future", well-known as a report of G.H. Brundtland, the definition of SD was given: "Sustainable development is the development that meets the needs of the present, but does not threatens the ability of future generations to meet their own needs" [Our Common Future,1989: 59]. This definition became most widely-spread after its actual 
acceptance at the UN Conference on Environment and Development in 1992 in Rio de Janeiro (UNCED). This definition expresses the deep essence of the new model (form) of human development, which, according to the opinion of authors, should not be interrupted by any global catastrophe of human origin.

In such a definition of SD the priority place is taken by the concept of necessity, which expresses the relation of the subject, i.e. a living rational being and the environment, both natural and social. However, as in the case of SD we actually speak about the whole mankind, we have in mind the need for natural conditions and resources of the Earth and the space surrounding the civilization. Emphasis is also placed on future generations, which is directly related to the change of generations and youth policy [Ilyinsky, 2006].

Because in the UDM (i.e. in modern model, so called at UNCED), it is impossible to satisfy equally both current and future generations, this model of development implies a rather fast dramatic finale of wasteful market-economocentric development of mankind. This tragic end of the "market mankind" (if modern economocentric orientation can be called so) is associated with anthropoecological catastrophe, first of all with the deterioration of the environment and depletion of natural resources. That is exactly why the satisfaction of needs of future generations expresses not yet existing at the moment, but future - a kind of leading humane need of the entire human race to continue its temporal prolongation (the "linear thinking" effect).

The majority of people living on the planet do not feel this "here and now" need if to consider the development of mankind in the coordinates of modern market economocentric element. However, such need for the continuation of the human race emerges if the need and the importance of ensuring the survival and the temporal prolongation of existence for indefinitely long future are realized. Thus there emerges a potential need for the formation of a new "youth strategy", in the appearance and existence of future generations, which in principle should exist for indefinitely long period of time. This need for the implementation of life support for future generations is beyond the momentary "market horizon" of thinking, it is directed towards the very distant humanistic future, and has a fundamentally virtual-strategic nature.

The discussed leading need for survival and the continuation of the human race will affect the modern needs, transforming them more efficiently in the direction of its future implementation. There is a contradiction between apparently superfluous needs of present generations and opportunities of satisfaction of the needs by future generations. The growth of needs of current generations, particularly unreasonable and pathological, leads to a significant reduction of opportunities and ways to meet the vital needs for natural resources and environmental conditions of future generations up to extinction of mankind as a result of, for example, anthropoecological catastrophe already at the beginning of the current millennium. It is a kind of a globally-temporal law of "conservation of energy": the more irrationally the present generations waste environmental resources, the less is left for future generations. In addition, not only resources but also environmental conditions that can be considered as resources in the broad synergetic understanding.

Such a perspective requires a change in the modern model of development of civilization for the purpose of the progressive realization of perceived leading need for the "continuation of humanism" for an indefinite future. As this need is already recognized (though it is not put on the priority place), then it is a human interest, of long-term strategic nature that more and more should be considered with the transition to a sustainable future. That emerged contradiction 
between the current and future needs can be solved only by the increasing of opportunities to satisfy the needs by future generations through reasonable restriction (without affecting the vital needs) to meet the needs of present generations. In conditions of the limitedness of planetary resources modern generations live, and do not realize it, borrowing at the expense of future generations, actually thoughtlessly wasting natural resources, and creating for them worse living conditions in the biosphere, which is obviously inhumane, bearing in mind the strategic perspective.

It is appropriate to call the process of increasing satisfaction of perceivable leading needs, which go beyond the short-term "market horizon", the process of futurization of satisfaction of needs (and interests). And the transition to SD suggests a long-term holistic system of activities that implement the process of rationalization and futurization and thus "strategic humanization" and "temporal optimization" of needs. This implies a gradual abandonment of the modern society of consumption and the transition to a more rational satisfaction of needs, or, as they also say, coevolutionary reasonable needs, which involves transition to SD, extending the temporary existence of mankind on the planet and in the Universe. In this case, there will occur the temporal optimization of the needs of present and future generations, which is manifested in futurization and rationalization of needs of mankind, which should be seen not just as a whole in the spatial sense, but as a whole in a temporal dimension. It is doubtful whether the purpose of the process of globalization is the achievement of only spatial integrity in UDM, which sooner or later will be destroyed by anthropoecological catastrophe. It is clear that such a goal in the strategic perspective looks anti-humane, which indicates the need to see the prospects of globalization at the perspective of transition to SD.

Transition to SD involves the formation of not only globally-spatial integrity, but also the deployment of a more systematic process - achievement of system-temporal integrity of mankind. This means the acquisition by mankind of its rational human future that threatens to lose the continuation of a modern model of socio-economic development. The acquisition of temporal integrity of civilization by mankind can occur only in the process of optimization and futurization of the needs of present and future generations. In this sense, we can agree with Nikolay Marfenin that "modern ideologies should be based on the choice between short-term and long-term benefit in nature management" [Marfenin, 2007: 599]. And although it is stated in relation to nature management, however it is his rationalization that underlies future SD as essentially a synonym for SD, to which Victor Danilov-Danil'yan draws attention [DanilovDanil'yan, 2003: 123-124] and about which it was already written many years ago [Ursul, 1993]. Of course, we are talking mainly about conceptual, from the positions common sense. interpretation of the future model of civilization exactly as a model of the survival of mankind, as a kind of "earthly invariant" of social level of evolution that occurred on our planet. The above means that you need to consider in shaping long-term strategy of development of civilization the most important insights of the general theory of evolution [Bazaluk, 2014; Bazaluk, 2015].

The first work (at least in Russia), which examines the relationship between environmental education and prospects for sustainable development (SD), published in Russia more than 20 years ago [Environmental education, 1993], to a large extent anticipated the basic ideas of "Strategy of the European Economic UN Commission for Education for Sustainable Development" adopted in 2005 in Vilnius by high-level meeting of representatives of the Ministries of Environment and Education.

Moreover, it becomes clear that the Education for Sustainable Development (ESD) is not only an assumption to achieve sustainable development, but a priority means of it, i.e. we 
can say that transition to a new course of the development of the civilization begins with the formation of education for the benefit of sustainable development, achieving the global dimensions. It should also be agreed with the opinion that ESD is the order of forwardlooking politicians who have a good sense of direction in megatrends of the world community [Marfenin, 2008: 298].

For most ESD researchers it is quite obvious that a new type of education is based on the environmental education [Ursul \& Demidov, 2004; Stepanov, 2009; Towards Education 2006]. During the research it has become clear that the environmental education in the unsustainable development model (i.e., that it represents currently) and something it should become in the sustainable development model — these are different types of education in terms of content and quality.

Despite the fact that the environmental education is, in a sense, an invariant of the modern education and its future system (model), which is adequate to the SD, within the latter it acquires some features caused by this new type of the civilization development.

\section{Features of Education for Sustainable Development}

Some meaningful features of this new type of the global education, which are the most significant, can be formulated now on the basis of the purposes and principles of the SD (sustainable development).

Firstly, if the environmental education for the benefit of the SD is guided by the purposes and principles of the SD, i.e. the salvation of mankind and the planet (biosphere), then it should contain the educational information building the future culture of the SD, i.e. not everything existing nowadays. It is not necessary anymore to argue that culture is evolving and will continue to evolve, although last century Leslie White had to defend the evolutionary approach to culture. However, it is important not so much the past of culture genesis as its future directed at transition to the SD. It seems that this transition will be coupled with the formation of a new type of the world culture, which is appropriate to refer as the sustainable development culture, which is gradually evolving in a noospheric culture during noospherogenesis [Ursul, 2003].

It is quite clear that a recovery from the global crises, and especially from socioenvironmental, is possible only on the basis of building of a fundamentally new "sustainable culture" associated with the new way of the relationship between man and nature, which exclude the possibility of widespread environmental damage. This future type of culture should also be connected with security culture, and not only of environmental security.

Secondly, the environmental education in the unsustainable development model seems alien to this model, it looks kind of a virtual "implant from the future". When the whole modern model of the functioning and development of a society has economic and centric character, showing in all areas the consumer orientated marketing, it does not accept the broad introduction of the environmental imperatives, rejecting everything that is associated with the restriction of receiving of profit and benefit. All other components of the education system of the unsustainable development model, according to the principles of the model, react and begin to "put pressure" on their own and at the same time "alien" component in the form of the environmental education. This contradiction between the interests of present and future needs cannot without difficulties and problems resolved in favor of the latter (the contradiction between the modern "economic" generations and future generations in a hypothetical society with the SD). 
A way out of this contradictory situation is seen in the fact that all other components of education: i.e. its economic, social, humanitarian, technical and technological and other components should also "work" for the transition to sustainable future. Development of the environmental education only, even applying more and more efforts and finances, will not be able to bring the educational system to a new level corresponding to the purposes and principles of the SD, especially in its noospheric direction. The SD is a system of economic, environmental, sociocultural, political, demographic and other aspects of human activity targeted at the survival of the civilization, and that should be reflected in innovative and advance "sustainable education".

The environmental imperatives are indeed critical within the understanding of the SD concept (especially if it comes from environmentalists). However, during the theoretical and methodological research it has become clear that the SD is not just adding the ecology to the traditional development, but is fundamentally new transformations in all areas of mankind development, i.e. it is different in essence "innovation revolution" on a global scale.

Unfortunately, the ESD is still based on a very simplified and one-sided concept of the SD, which is not adequate enough, as the environmental aspect and its relationship with economy and social sphere are mainly emphasized. Certainly, it is necessary to do, but this is not enough, it is important to expand the subject field of the study of the stability issue, and to make the SD concept more integral.

The environmental emphasis of this concept was the right step, focused on a long-term, strategic perspective. It is important to proceed from the assumption that the biosphere, as noted by Vladimir Vernadsky, is that earth shell, "where life can only exist. A human being, according to its nature, is inseparable from it. And this non-separability begins only now to reveal truly in front of us" [Vernadsky, 1965: 324]. Therefore, addressing the issue of the human environment meets the requirement of a scientist to consider the socioeconomic development in conjunction with this environment.

Taking into account this requirement, the sustainable development implies survival of the civilization and even improvement of the life quality of the world population without increasing in usage of natural resources and without degradation of the environment to such an extent that it would not result in exceeding of the carrying capacity of the Earth as an environmental system. Despite the fact that the transition to the sustainable development may require taking different actions in each state, the current efforts to build a sustainable future require an integrated approach to activities in three key areas : economic, social and environmental. Formulation of a new development strategy means gradual integration into a single self-organizing system of economic, environmental and social fields. In this regard, the SD should be characterized by (at least) economic efficiency, biosphere compatibility and social justice under general decline in anthropogenic impact on the biosphere.

Thus, as mentioned above, the new model of the civilization development has turned out, on the one hand, to be more promising, as with its help the civilization will be able to survive. However, on the other hand, created only in theory, this model is less systematic and does not take into account many components in terms of the interrelation of development and security, which characterize the modern development model.

Thirdly, the environmental education in the unsustainable development model (as well as all other types and forms of education) is far behind from the real life and from the research frontier. Of course, not all environmental education can be characterized as "lagging behind"; it also has anticipated features. However, being in the integral system of the "unstable" 
global education, currently the environmental education cannot in the proper degree be such anticipated education, which would be adequately consistent with the importance of resolving of the environmental issues and transition to the SD.

Meanwhile, the forward-looking features of the environmental and other types of education, as has often been emphasized, follow from the fact that the environmental (especially global) issues and their negative and disastrous consequences can be only prevented, i.e. their solution is fundamentally proactive. There will be no one to eliminate the consequences of a planetary socio-environmental or any other planetary disaster, so the only way to secure from it is to take anticipated decisions and actions to prevent it globally. Within the unsustainable development model the most common way to respond to emergencies, crises and disasters is the elimination of their consequences (when they are local), but within the new civilization model the priority and dominant way should prevent them (which, by the way, is much cheaper in economic terms). And it requires the formation, to a certain extent, of a "anticipated culture", and it is possible not in all cultural fields (because some of them are basically focused on the past).

Fourthly, the environmental education, as, indeed, and all environmental activities throughout the world, after the Stockholm Conference on the Environment under the auspices of the United Nations, focused its attention mainly on local environmental issues. Improvement of the environmental situation in a particular ecosystem, for example in a particular pool or city, is important, but this approach is typical for the solution of environmental issues in the unsustainable development model. Inspired by the solution of local environmental issues, the international community after two and even more so after three decades after the Stockholm Conference revealed that the global environmental situation had not improved, but deteriorated significantly. And this despite the fact that only during these three decades the enormous sums, perhaps exceeding several trillion USD, were spent on the local environmental actions all over the world. This amount is still increasing, and the global environmental situation and its prospects are declining because the emphasis is still on local environmental activities, and all this is typical exactly for the unsustainable development model [GEO -5, 2012].

In addition, even though the Club of Rome in due time encouraged to think globally and act locally, this appeal was understood primarily in terms of performance of local activities. Nobody even thought, holding local activities, about simultaneous improvement of the global environmental situation. Now, referring to the prospects for a sustainable future and following Vladimir Vernadsky, it is necessary both think and act globally and locally, without breaking their interrelation into the mental and practical components. The environmental degradation on the planetary scale due to the majority of the local environmental events has occurred, resulting from the fact that due to their holding the human pressure on the biosphere has been not decreased, but has been increased (because for their implementation the resources are used and environmental degradation occurs in other parts of the planet). Let us say, to clean a pool or river it is necessary to use energy and other resources taken from other places from other ecosystems, which leads to their disruption and pollution. All of them are summed up at the biospheric global level, and as a result the local events appear as "sweeping trash under the bed" and approach a planetary ecocatastrophe. Moreover, due to synergetic reasons there are more degrading areas on the planet, and this is reflected on a planetary level more and more anticipating and intensifying a socio-ecological crisis and approaching a global ecocatastrophe.

That is why it is important during any environmental activities to ensure that the global environmental situation would not be worsened and even better if the human pressure on 
the biosphere would be decreased due to the whole complex of environmental measures aimed at implementing the purposes of the SD. The transition to this type of development has a fundamentally planetary character by virtue of the integrity and strong environmental interrelations of the biospheric components, and the globalization of social activities. Global security in terms of the environment or any other is of higher priority than the security of any part of the biosphere and sociosphere. And it is clear why: in case of a global environmental disaster, all local improvements of ecosystems will be negated, the national security (including environmental as well) of any state will be destroyed, and safety of an individual, society and any other communities is out of the question at all. Natural ecosystems - biogeocenoses and in the first place biota, especially in its higher level — will suffer as well.

All the above mentioned priorities should be fully taken into account in the environmental education, which will become an integral part of the ESD. It is possible to come up with other arguments, which supports the fact that the environmental education before the SD strategy and after its appearance is fundamentally different forms of education. It is important to realize this and direct the vector of further development of the environmental education towards the transition of the world community to the SD, the ultimate purpose of which is the establishment, in the global scale, of the most secure and highly intelligent society — the noosphere.

\section{Conclusions}

The ESD will substantially include the environmental component both in the form of ecologization of education and professional environmental education. In addition, supporters of this point of view consider the process of establishing of this type of education as a transition from the environmental education to the ESD, as it emerges in the aforementioned Strategy UN Economic Commission for Europe. A new recently adopted Global Program of Action on Education for Sustainable Development opens up even broader perspectives [Global Action, 2014].

Taking the environmental interpretation of the ESD as a basic, however it is important to note the trend to expand the understanding of this type of education at the expense of other SD system components. It has become clear that this future type of development will consist of at least several of its components: social sustainable development, economic sustainable development, demographic sustainable development and environmental sustainable development, and so forth. All these and other components of a single socionatural sustainable development become persistent types of development if they are subject to certain limitations and all of them are all linked in a single system, which differs from the one-dimensional economic and centric unsustainable development, mainly characterized by economic efficiency.

Along with this, let us call it "system-conceptual" interpretation of this type of development, an understanding has become to form recently about more secure development than the existing one and not only in environmental terms. In fact, the identification of the connection of the SD with the issue of environmental security, as it has turned out, has come down to the definition of those restrictions and, consequently, new rules, which express the maximum allowable (carrying) capacity of the ecosystems and the biosphere altogether. The SD is such a regulatory type of development that occurs within the carrying capacity of the ecosystems, i.e. in this case the normalization is of natural kind. However, this understanding expresses mainly its environmental aspect and is positioned as an environmentally friendly development with 
the following from this norms, principles and standards.

However, in case of consideration not only environmentally friendly, but also other forms and types of safe development, certain boundaries (limits) and standards of safe (in some way or another) development can be defined. It is subject to economically safe development, social, informational, political, technical and all other forms and types of development and the corresponding forms of security. The development of any system as a whole is sustainable, if it occurs within the appropriate "regulatory security corridor", i.e. this or that "carrying capacity" of human activities.

In this sense, the environmental security is not different from other types of security, but it is important that the concept of the SD was first formulated only in connection with the environment. And before the awareness of the connections between the concepts "development and the environment", other types of safety were studied along with the environmental, but the concept of the SD was created only through the environmental understanding of development and, as it became clear, especially through environmentally friendly development. Only later it became clear that in addition to the environmental security, it is important to include other characteristics of a real development process, i.e. its economic, political, social, demographic, information dimension, and so forth. However, with such a system synthesis, it is not very clear why we should include only those characteristics that are associated with the appropriate kind of security: economic, demographic, social, and so forth. It was an unexplainable "error" of conceptually theoretical awareness of the SD issues, and it is important to adjust it in a new, more systematic concept of this type of development in its broader "safe" interpretation.

In general, the concept of the SD could appear not in the "environmental and centric" form, if it had been realized that it was necessary to change the course of development of the world community due to other circumstances. Although historically it first came through environment, yet a new type of development cannot be associated only with the implementation of the strategic environmental imperatives and priorities. The SD is after all safe and innovative type of global development in all respects, which is implemented in a fairly narrow evolutionary corridor (within the carrying capacity of the ecosystems). Therefore, at a certain level of "normative" understanding of the essence of this type of development, it is important to state that it is characterized by globality, systematicity, continuity, security and adoption of forwardlooking solutions. The ESD model, of course, will more and more include security issues [Ursul, 2009; Ilyin \& Ursul, 2016].

Therefore, understanding of the ESD issues should not necessarily occur only through environment and environmental security. This type of development can be interpreted also through security issues and through directions of social activity (including security) included in the SD system, which have previously been studied as relatively autonomous forms of human activity. As the most cardinal and priority characteristics of SD are identified, they should be mastered by education in the interests of sustainable development, joining existing ESD components, and thereby a system-evolutionary synthesis of this new perspective form of the educational process will be carried out. For example, you can enable this advanced educational system becoming cosmic education, which also will go to sustainable development path [Bazaluk, 2013; Bazaluk \& Blazhevich 2013].

It is clear that both the SD and education for this type of the civilization process should be systemic in nature and should be applied to all educational disciplines and courses, and, first of all, should be implemented where there are more reasons to include the SD ideas in education. It is important to develop the ESD in various directions, including environmental, "safe" and 
other paths forming the system and integral concept and, in the long term, the SD theory.

The future science, on which the education is based, emerges as a totally new stage of development of single world science, which corresponds with the establishment period of the sphere of mind (noosphere) through the SD with a distinct emphasis on the study of the global development and the awareness of the future of mankind and all creation. The noospheric science, following the post-non-classical science, altogether with education will form in the long term a single scientific and educational process, forming planetary and integral noospheric consciousness both of an individual and all mankind, which advances practical global activities and contributes to its optimal development within coevolution with the nature of the Earth and Universe.

\section{[D] References}

Bazaluk, Oleg. Postmodernism: Philosophy of Education. Future Human Image. 2 (5), 2015: 9-22.

Bazaluk, Oleg. The Strategies of Systematization of the Theories of Education. The Main Meaning and Features of the Theories of Education of Plato's and Isocrates' Lines. Future Human Image. Volume 7, 2017:11-27.

Bazaluk, Oleg. Plato's and Isocrates' Traditions in the Development of Educational Theories in the History of Culture. Annals of the University of Craiova - Philosophy Series, Nr. 40 (2/2017): 5-18.

Bazaluk, Oleg. The Feature Transformations of the Basic Meanings of Greek Paideia in the Educational Theories in the Middle Ages. $\mathrm{XX} \Lambda \mathrm{H}$ 12.1, 2018: 243-258.

Bazaluk, Oleg. The Theory of Evolution: From a Space Vacuum to Neural Ensembles and Moving Forward. Cambridge Scholars Publishing, 2016.

Bazaluk, Oleg, and Tamara Blazhevich. Cosmic Education: Formation of a Planetary and Cosmic Personality. Philosophy and Cosmology. Volume 11, 2013: 147-160.

Danilov-Danilyan, Viktor. Sustainable Development (Theoretical and Methodological Analysis). Economics and Mathematical Methods. Vol. 39. No 2, 2003:123-138 (in Russian).

Education for Sustainable Development. Edited by Nikolay Casimov. Moscow-Smolensk: Universum, 2004 (in Russian).

Environmental Education and Sustainable Development. Edited by Akady Ursul. Moscow: Luch, 1996 (in Russian).

GEO -5. Global Environment Outlook. Summary for Policymakers. Nairobi: UNEP. 2012.

Global Action Programme on Education for Sustainable Development. https:/en.unesco.org/ gap (accessed 17 November 2017).

Ilyin, Ilya, and Arkady Ursul. Education, Society, Nature: the Evolutionary Approach and Global Perspective. Moscow: Moscow University Press, 2016 (in Russian).

Ilyinsky, Igor. Between Past and Future. Moscow: MosGU. 2006 (in Russian)

Marfenin, Nikolay. Sustainable Development of Mankind. Moscow: Moscow University press, 2007 (in Russian).

Marfenin, Nikolay. The Scientific Basis of Education for Sustainable Development. To Necessary Outlines of Future Civilization. Edited by Aleksey Nikitin \& Stanislaw Stepanov. Moscow: MNEPU. 2008 (in Russian).

Our Common Future. World Commission on Environment and Development Review. 
Translation from English. Moscow: Progress. 1989 (in Russian).

Stepanov, Stanislaw. Globalization, Sustainable Development, Education. Conceptual Foundations of Environmental Education in Higher Education for Sustainable Sevelopment. Moscow: MNEPU, 2009 (in Russian).

Towards Education for Sustainable Development in Russia. Edited by Nikolay Casimov \& Svetlana Malkhazova. Moscow: Moscow State University, 2006 (in Russian).

Ursul, Akady. Culture, Education and Safety in the New Paradigm of Development. Synergetic Paradigm. Man and Society in Conditions of Instability. Moscow: RAGS. 2003 (in Russian).

Ursul, Akady. Way to the noosphere. The Concept of Sustainable Development and the Survival of Civilization. Moscow: Luch. 1993 (in Russian)

Ursul, Akady. The National Security Strategy of Russia and Education for Sustainable Development. Open Education. No 4, 2009: 34-48 (in Russian).

Ursul, Akady, and Fedor Demidov. Education for Sustainable Development: the Scientific Basis. Moscow: RAGS, 2004 (in Russian).

Ursul, Akady, and Tatiana Ursul. Education and Globalistics. Future Human Image. Volume 7, 2017: 136-153.

Vernadsky, Vladimir. The Chemical Structure of the Earth's Biosphere and its Environment. Moscow: Nauka, 1965 (in Russian). 